\title{
AVALIAÇÃO DE PARÂMETROS HIDRÁULICOS E MANEJO DA IRRIGAÇÃO POR MICROASPERSÃO EM ÁREA DE ASSENTAMENTO ${ }^{1}$
}

\author{
FÁBIO S. SANTIAGO², ABELARDO A. A. MONTENEGRO ${ }^{3}$, \\ SUZANA M. G. L. MONTENEGRO ${ }^{4}$
}

\begin{abstract}
RESUMO: Este trabalho foi desenvolvido em solo aluvial do semi-árido de Pernambuco, suscetível à salinização, visando a orientar agricultores de assentamento para instalar, avaliar e manejar racionalmente sistemas de irrigação por microaspersão, para atender ao plantio da cultivar de repolho Midori. Adotando-se percentagem de área molhada igual a $100 \%$, foram avaliados nos setores da unidade operacional a variação de vazão e pressão, os coeficientes de uniformidade (CU) e a eficiência de aplicação (EA). A uniformidade da irrigação apresentou valores elevados, superiores a 93\%. A eficiência de aplicação foi boa, com valores maiores que $80 \%$. O sistema foi então utilizado na irrigação, utilizando como controle o tanque "Classe A", durante um período de 75 dias, e conduzido por um agricultor local. Considerando os riscos de salinização na área, promoveu-se lavagem contínua do solo. Foi adotado um coeficiente de lixiviação médio de 1,21, com desvio-padrão de 0,45 , para o período sem precipitação. Praticamente em todo o experimento, o sentido do gradiente de potencial total da água do solo foi ascendente, com média de 1,14 e 0,21 de desvio-padrão. Preveniu-se com isso a ocorrência de ascensões capilares, estabelecendo-se um perfil drenante, bem como se manteve a condutividade elétrica da zona saturada em equilíbrio. Não foi observada a saturação do solo na zona radicular.
\end{abstract}

PALAVRAS-CHAVE: aluviões, microaspersão, manejo de salinidade.

\section{HYDRAULIC PARAMETERS EVALUATION AND MANAGEMENT OF MICROSPRINKLER IRRIGATION IN SETTLEMENT AREA}

SUMMARY: This study has been carried out in alluvial area in the semi-arid zone of Pernambuco State, Brazil, subject to salinisation, aiming to guide local farmers to install, evaluate and to manage micro sprinkler irrigation systems, raising Midori cabbage cultivar. Adopting the area percentage of wetted soli $100 \%$ the sub units have been evaluated regarding the discharge and pressure variation, and also coefficients of uniformity (CU) and efficiency of application coefficient (EA). The irrigation uniformity was high, greater than $93 \%$. The efficiency of application coefficient could be classified as good, exhibiting values greater than $80 \%$. The system was then used to local scale irrigation, adopting a "Class A" tank as a management control, during a 75 days period and conducted by a local farmer. Continuous leaching has been implemented throughout the study, for salinity control. Average leaching coefficient equal to 1,21 with standard deviation of 0,45 has been adopted, for the period with no rainfall. Upward gradients have prevailed, with average of 1,14 and 0,21 standard deviation, preventing capillary flow to occur. A stable electrical conductivity in the saturated zone has been observed. Soil saturation has not been recorded in the root zone.

KEYWORDS: alluvial areas, micro sprinkler, salinity management.

\footnotetext{
${ }^{1}$ Extraído da Dissertação de Mestrado do primeiro autor.

${ }^{2}$ Eng ${ }^{\circ}$ Agrônomo, Especialista em Conservação do Solo, Mestre em Agronomia, CAMIPEC, BR 232, km 421, Serra Talhada - PE, email: fabioirriga@bol.com.br

${ }^{3}$ Prof. Adjunto, Ph.D., Depto. de Tecnologia Rural, Área de Recursos Hídricos, UFRPE, Recife - PE, e-mail: monte@ hotlink.com.br

${ }^{4}$ Prof. Adjunto, Ph.D., Depto. de Engenharia Civil, Área de Recursos Hídricos, UFPE, Recife - PE, e-mail: suzanam@ufpe.br

Recebido pelo Conselho Editorial em: 12-2-2003

Aprovado pelo Conselho Editorial em: 23-7-2004
} 


\section{INTRODUÇÃO}

A expansão da agricultura irrigada tem-se tornado preocupante, devido ao elevado consumo e às restrições de disponibilidade de água, bem como ao risco de degradação do sistema solo-água-planta.

Segundo BERNARDO (1997), as baixas eficiência e uniformidade dos sistemas de irrigação, e a limitada lixiviação de sais do solo estão dentre os principais fatores que influem na degradação do solo. Em áreas de agricultura familiar, o cenário de risco de degradação se agrava, devido ao limitado nível de capacitação dos agricultores.

De acordo com o IBGE (1996), os agricultores familiares representam 85,2\% do total de estabelecimentos rurais no Brasil, ocupando 30,5\% da área total e sendo responsáveis por 37,5\% do valor bruto da produção agropecuária nacional, embora recebendo apenas $25,3 \%$ do financiamento destinado à agricultura e sendo carentes de assistência técnica e de capacitação. A região Nordeste é a que apresenta o maior número de agricultores familiares.

A uniformidade de irrigação interfere diretamente nos processos de infiltração. RIBEIRO et al. (2003) destacam que, após a redistribuição da lâmina aplicada no perfil do solo, fluxos de infiltração podem ser substituídos por ascensões capilares, em presença de lençol freático raso, e em áreas com elevadas demandas evaporimétricas. Tais inversões reduzem a eficiência da lixiviação e podem incrementar a salinidade do solo. Conforme AYERS \& WESTCOT (1999), o manejo da salinidade deve incluir tanto a drenagem, como a lixiviação, necessária para evitar a acumulação excessiva de sais no perfil, em particular em áreas aluvionares do semi-árido. De acordo com LIBARDI (2000), há carência de estudos da dinâmica da água em condições de campo, com ênfase nos fluxos na zona radicular e em condições não-saturadas. Para avaliação de tais fluxos, as propriedades hidráulicas da zona não-saturada precisam ser estimadas. Dentre os métodos de campo mais utilizados para medir as propriedades hidráulicas não-saturadas, destaca-se o permeâmetro de Guelph (REYNOLDS et al., 1985).

BERNARDO (1996) ressalta que, mesmo na microaspersão, para a qual se tem melhor controle da lâmina aplicada, é recomendado após a instalação e durante a vida útil do sistema, verificar a uniformidade de irrigação, particularmente nos sistemas sem aplicadores compensados, como, por exemplo, naqueles utilizados na agricultura familiar. BARRETO FILHO et al. (2000) recomendam os métodos de MERRIAN \& KELLER (1978) e da ASAE (1996) para a avaliação do coeficiente de uniformidade (CU) na irrigação localizada. Conforme ABREU et al. (1987), a desuniformidade de aplicação pode ser resultado de diferenças de pressão na rede, de perdas de carga excessivas, de irregularidades topográficas, bem como de insatisfatória uniformidade de fabricação dos emissores.

No assentamento localizado na área aluvionar da Fazenda Nossa Senhora do Rosário, Pesqueira$\mathrm{PE}$, as 63 famílias assentadas vêm recebendo treinamentos sistemáticos. Um sistema-piloto de microaspersão para cultivo de hortaliças foi implantado, de modo a permitir o uso racional da água, o qual vem sendo operado pelos próprios assentados, sob supervisão de um técnico.

Os objetivos deste trabalho foram avaliar os parâmetros hidráulicos de um sistema de irrigação por microaspersão, bem como os parâmetros de manejo visando à otimização e sustentabilidade do uso da água na agricultura familiar, detalhando a dinâmica da água na zona radicular.

\section{MATERIAL E MÉTODOS}

A área de estudo está localizada na Fazenda Nossa Senhora do Rosário, a 230 km de Recife-PE, nas proximidades da rodovia BR-232, com coordenadas geográficas de $08^{\circ} 10$ ' $25^{\prime \prime} \mathrm{S}$ e $35^{\circ} 11^{\prime} 25^{\prime \prime} \mathrm{W}$, de latitude e longitude, respectivamente, e $650 \mathrm{~m}$ de altitude. O clima do local é classificado, segundo Köeppen, como BShw' semi-árido quente, caatinga hiperxerófila, com temperatura média anual em 
torno de $27^{\circ} \mathrm{C}$ e precipitação média anual de $600 \mathrm{~mm}$. A umidade relativa média anual do ar é de $73 \%$, e a velocidade média do vento é de $2,5 \mathrm{~m} \mathrm{~s}^{-1}$ (CISAGRO, 1990).

\section{Avaliação de parâmetros físicos e hidráulicos do solo}

A condutividade hidráulica saturada da área foi avaliada por MONTENEGRO (2000), utilizando permeâmetro de laboratório, em $9,58 \mathrm{~mm} \mathrm{~h}^{-1}$. Segundo GARDNER (1958), onde a saturação não é atingida, a condutividade hidráulica pode ser expressa em função do potencial matricial por:

$$
\mathrm{K}\left(\Psi_{\mathrm{m}}\right)=\mathrm{K}_{0} \exp \left(-\alpha \Psi_{\mathrm{m}}\right)
$$

em que,

$\mathrm{K}_{0}$ - condutividade hidráulica saturada, e

$\alpha$ - constante dependente do meio poroso (BRESLER, 1978), a qual descreve a inclinação de $\ln \left(\mathrm{K}\left(\Psi_{\mathrm{m}}\right)\right)$ vs. $\Psi_{\mathrm{m}}$ (potencial matricial).

Os valores de $\alpha$ para solos arenosos e franco-arenosos são da ordem de 10 a $20 \mathrm{~m}^{-1}$, de 1 a $2 \mathrm{~m}^{-1}$ para solos francos, e de 0,1 a $0,2 \mathrm{~m}^{-1}$ para solos argilosos.

De modo a possibilitar a aplicação da eq.(1), foi utilizado um permeâmetro tipo Guelph (REYNOLDS et al., 1985). A condutividade hidráulica saturada foi estimada em $9,20 \mathrm{~mm} \mathrm{~h}^{-1}$, com constante $\alpha$ igual a $8,97 \mathrm{~m}^{-1}$.

SANTIAGO (2002) determinou a curva característica do solo, a partir de amostras indeformadas de solo no extrator de Richards, de acordo com procedimentos da EMBRAPA (1979), obtendo valores de 0,$22 ; 0,07 ; 0,44$ e 0,$37 ; 0,10 ; 0,44\left(\mathrm{~cm}^{3} \mathrm{~cm}^{-3}\right)$, para as umidades na capacidade de campo, no ponto de murcha permanente, e para a porosidade total do solo, de 0 a $60 \mathrm{~cm}$ e de 60 a $90 \mathrm{~cm}$, respectivamente. Também foi determinada a distribuição granulométrica do perfil do solo, segundo a metodologia da EMBRAPA (1997), na estação tensiométrica utilizada para o monitoramento dos potenciais de água no solo, obtendo-se porcentagem de areia de $26,74 \%$ e $33,56 \%$, e porcentagens de argila de $34,07 \%$ e $31,57 \%$, para as camadas de 0 a $60 \mathrm{~cm}$ e de 60 a $90 \mathrm{~cm}$, respectivamente. Nessa mesma locação, o perfil do solo foi texturado a tato até a profundidade de $3 \mathrm{~m}$, não se detectando camada de impedimento.

\section{Desempenho do Sistema de irrigação}

Foi dimensionado e implantado pelos autores desse estudo um sistema-piloto de microaspersão para hortaliças, cuja unidade operacional é composta de 3 setores, apresentando como fonte de água um poço tipo Amazonas, cuja vazão média é de $4,5 \mathrm{~m}^{3} \mathrm{~h}^{-1}$. O microaspersor escolhido foi da marca Carborundum, sem compensação de pressão, de bocal verde-claro e com diâmetro igual a 1,0 mm, bailarina cinza e diâmetro molhado igual a $6,2 \mathrm{~m}$, vazão de $43 \mathrm{~L} \mathrm{~h}^{-1}$ para uma pressão de operação de $150 \mathrm{kPa}$, gerando uma intensidade de aplicação de $3,58 \mathrm{~mm} \mathrm{~h}^{-1}$, para espaçamento de $4 \mathrm{~m} \mathrm{x} 3 \mathrm{~m}$. Os setores $1 ; 2$ e 3 possuem área de $1.296 \mathrm{~m}^{2}$, e as linhas laterais de polietileno têm comprimento de $48 \mathrm{~m}$ e diâmetro de $16 \mathrm{~mm}$, e as de derivação de PVC, com diâmetro de $50 \mathrm{~mm}$ (PN40). As linhas mestra e adutora foram dimensionadas em PVC com $50 \mathrm{~mm}$ (PN80). Adotou-se porcentagem de área molhada de $100 \%$. A cultivar de repolho Midori (Brassica oleracea var. capitata) foi implantada com espaçamento de $0,6 \mathrm{~m} \times 0,6 \mathrm{~m}$.

Foram medidas as variações médias de pressão e vazão ao longo das linhas laterais, segundo amostragem-padrão, especificamente na primeira linha lateral (início da derivação), na quarta (a 1/3 do início), na oitava (a $2 / 3$ do início) e na décima segunda (final da derivação). Os emissores de cada lateral foram escolhidos segundo o mesmo critério-padrão de distribuição: primeiro emissor, emissor a $1 / 3$ do comprimento da lateral, emissor a $2 / 3$ da lateral e último emissor da lateral. Para a avaliação da vazão, foi utilizado procedimento volumétrico. Realizaram-se também as medidas de pressão, 
utilizando um manômetro portátil. Foram determinadas as médias das variações máximas de pressão $\varepsilon_{\mathrm{P}}$ e de vazão $\varepsilon_{\mathrm{Q}}$ ao longo das linhas de distribuição e nos setores irrigados, em relação ao máximo valor medido, conforme equacionamento apresentado por BARRETO FILHO et al. (2000).

Utilizando-se dos dados de vazões e pressões encontrados em cada setor, foram determinados os coeficientes de uniformidade (CU) de irrigação e de pressão (CUP), de acordo com os métodos de MERRIAN \& KELLER (1978) e da American Society of Agricultural Engineers (ASAE).

No primeiro método, a uniformidade foi avaliada por meio de:

$$
\mathrm{CU}=100 \frac{\mathrm{q}_{25 \%}}{\mathrm{q}_{\mathrm{med}}}
$$

em que,

CU - coeficiente de uniformidade de irrigação do setor, \%;

$\mathrm{q}_{25 \%}$ - vazão média dos $25 \%$ de microaspersores com as menores vazões, $\mathrm{L} \mathrm{h}^{-1}$, e

$\mathrm{q}_{\mathrm{med}}$ - média das vazões coletadas nos microaspersores do setor, $\mathrm{L} \mathrm{h}^{-1}$.

Com os dados de pressão, avaliou-se o coeficiente de uniformidade de pressão a partir de:

$$
\mathrm{CUP}=\left(\frac{\mathrm{P}_{25 \%}}{\mathrm{Pm}}\right)^{\mathrm{X}} 100
$$

em que,

CUP - coeficiente de uniformidade de pressão no setor, \%;

$\mathrm{Pm}$ - pressão média do setor, $\mathrm{kPa}$;

$\mathrm{P}_{25 \%}$ - pressão média dos $25 \%$ de microaspersores com as pressões mais baixas no setor, $\mathrm{kPa}$, e

$\mathrm{X}$ - expoente de descarga do emissor no setor, que pode ser avaliado experimentalmente, conforme detalhado por MERRIAN \& KELLER (1978), e sugerido por BARRETO FILHO et al. (2000).

A eficiência de aplicação (EA) da unidade operacional foi estimada, conforme apresentado por MERRIAN \& KELLER (1978), como:

$$
\mathrm{EA}=0,9 \mathrm{CU}
$$

No Método da American Society of Agricultural Engineers (ASAE), a uniformidade de irrigação é baseada em coeficiente de uniformidade estatístico dos setores da unidade operacional, a partir de:

$$
\mathrm{CUE}=(1-\mathrm{CVT}) 100
$$

em que,

CUE - coeficiente de uniformidade estatístico de irrigação, e

CVT - coeficiente de variação total de vazão no setor, baseado no desvio-padrão da vazão e na vazão média dos setores, segundo discutido por BARRETO FILHO et al. (2000).

Esses mesmos autores sugerem a aplicação do coeficiente de variação por causas hidráulicas $\mathrm{CVH}$, com base no desvio-padrão das pressões dos microaspersores escolhidos segundo amostragempadrão.

De acordo com BRALTS \& KESNER (1983), o coeficiente de variação estatístico pode ser estimado como:

$$
\mathrm{CVE}=\sqrt{\mathrm{CVT}^{2}-\mathrm{X}^{2} \mathrm{CVH}^{2}}
$$


Segundo a ASAE (1996), tem-se "uniformidade excelente" para valores de CUE e CU entre 95 e $100 \%$, e 94 e $100 \%$, respectivamente, enquanto se considera a uniformidade como "boa" para valores de CUE e CU entre 85 e $95 \%$, e entre 87 a 94\%, respectivamente.

\section{Manejo da irrigação}

O manejo foi desenvolvido diariamente, utilizando o tanque "Classe A" para a estimativa da evapotranspiração de referência. O coeficiente de tanque (Kp) foi obtido segundo DOORENBOS \& PRUITT (1977), conforme condições locais.

Foram considerados 4 estágios de desenvolvimento para a obtenção dos coeficientes de cultura (Kc) do repolho. O $1^{\underline{o}}, 2^{-}, 3^{\circ}$ e $4^{\circ}$ estágios foram aplicados nos intervalos de 01-09-2001 a 26-9-2001

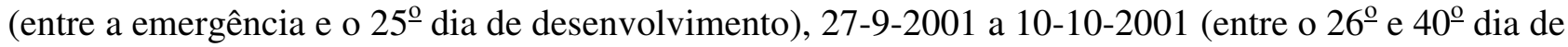

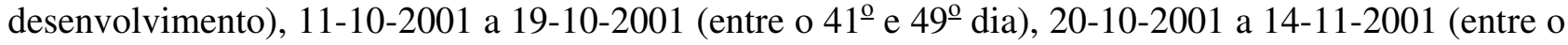
$50^{\circ}$ e $75^{\circ}$ dia), com valores iguais a 0,$70 ; 0,95 ; 0,90$ e 0,80 , respectivamente, conforme recomendado por DOORENBOS \& KASSAN (1979). As lâminas brutas para um turno de rega diário foram estimadas como:

$$
\mathrm{L}_{\text {bruta }}=\frac{(\mathrm{ETR}-\mathrm{P})}{\mathrm{EA}} \mathrm{C}_{\mathrm{x}}
$$

em que,

$\mathrm{L}_{\text {bruta }}$ - lâmina bruta diária ou total de irrigação, mm;

ETR - evapotranspiração real diária da cultura, mm, considerando coeficientes de tanque e de cultivo;

EA - eficiência do sistema de irrigação;

$\mathrm{C}_{\mathrm{x}}$ - coeficiente de lixiviação necessário para a manutenção do equilíbrio salino no perfil de solo, e

$\mathrm{P}$ - precipitação, mm, medida diretamente em pluviômetro instalado na área.

O coeficiente de lixiviação Cx foi calculado como (AYERS \& WESTCOT, 1999):

$$
\mathrm{C}_{\mathrm{x}}=\left(1-\left(\mathrm{CE}_{\text {irrig }} / \mathrm{CE}_{\mathrm{dre}}\right)\right)^{-1}
$$

De modo a estabelecer um plano de manejo prévio para o agricultor, cujas variáveis fossem apenas a evaporação do tanque e a precipitação lidas, adotou-se, para o cálculo do coeficiente de lixiviação, a condutividade elétrica da água de irrigação $\left(\mathrm{CE}_{\text {irrig }}\right)$ como uma média de valores históricos do período de setembro de 1997 a novembro 2000, estimada em 1,40 dS m${ }^{-1}$. Posteriormente ao experimento, verificou-se a adequação do valor adotado, tendo-se medido uma condutividade elétrica média real de $1,37 \mathrm{dS} \mathrm{m}^{-1}$ no período, próxima à estimada. Sugeriu-se um coeficiente de lixiviação igual a 2,0, nos dias sem precipitação pluviométrica, de modo a produzir condutividade elétrica de água de drenagem $\left(\mathrm{CE}_{\mathrm{dre}}\right.$ ) de 2,80 dS m${ }^{-1}$ (próximo à capacidade de campo e na parte inferior da zona radicular), de acordo com AYERS \& WESTCOT (1999). Este valor é compatível com o limiar de $1,80 \mathrm{dS} \mathrm{m}^{-1}$ para o extrato saturado do solo, para $100 \%$ de produtividade do repolho, segundo MASS \& HOFFMAN (1977). Diariamente, mediam-se o volume aplicado através de um hidrômetro e o tempo inicial e final de cada aplicação. A lâmina aplicada foi estimada através da razão entre o volume aplicado e a área de cada setor.

O potencial matricial foi monitorado através de uma bateria de tensiômetros com manômetro de mercúrio, instalados nas profundidades de $20 \mathrm{~cm}, 30 \mathrm{~cm}, 40 \mathrm{~cm}, 60 \mathrm{~cm}, 80 \mathrm{~cm}, 100 \mathrm{~cm}$ e $120 \mathrm{~cm}$. Os fluxos foram estimados através a lei de Darcy, convencionando-se como positivos os gradientes ascendentes. Para estimar o fluxo entre profundidades monitoradas, adotou-se a média aritmética das tensões matriciais para avaliar a condutividade hidráulica não-saturada. Os fluxos de entrada, na superfície do solo, são representados pela soma da precipitação e da irrigação, enquanto os fluxos de 
saída, pela evapotranspiração da cultura. Para avaliar o efeito da irrigação nos níveis de água e de salinidade do lençol freático subjacente, monitoraram-se dois poços de observação, um adjacente à estação tensiométrica e outro situado acerca de $100 \mathrm{~m}$ de distância e fora da área irrigada (adotado como controle).

\section{RESULTADOS E DISCUSSÃO}

\section{Desempenho do sistema de irrigação}

Um valor médio de 44,54 $\mathrm{L} \mathrm{h}^{-1}$ para vazão (Tabela 1) foi encontrado na unidade operacional, $3,45 \%$ maior que a vazão de $43,0 \mathrm{~L} \mathrm{~h}^{-1}$ recomendada pelo fabricante do emissor, quando operando com pressão de serviço de $150 \mathrm{kPa}$. Entretanto, para atingir esta vazão, foi estabelecido um valor médio de pressão igual a $175,60 \mathrm{kPa}$. Segundo o fabricante, este valor médio de pressão encontrado produz uma vazão de $46,64 \mathrm{~L} \mathrm{~h}^{-1}, 4,5 \%$ maior que a média determinada na unidade operacional.

A maior variação de pressão foi encontrada no setor 2, com valor igual a 7,91\%, enquanto, para linhas laterais analisadas individualmente, o setor 3 é o que apresenta maior variação, com valor de 10,38\% (Tabela 1). A variação média de pressão nas linhas laterais foi de 7,93\%, inferior ao máximo recomendado por KELLER \& KARMELI (1975), de 11\%. As linhas laterais atenderam a essa recomendação.

TABELA 1. Variação de pressão (P) e vazão (Q) nos setores irrigados por microaspersão.

\begin{tabular}{lrrrr}
\hline \multicolumn{1}{c}{ Variáveis } & Setor 1 & Setor 2 & Setor 3 & \multicolumn{1}{c}{ Média } \\
\hline Pressão $(\mathrm{kPa})$ & 179,72 & 180,83 & 166,20 & 175,60 \\
Vazão $\left(\mathrm{L} \mathrm{h}^{-1}\right)$ & 45,46 & 46,15 & 42,03 & 44,54 \\
$\mathcal{E}$ Pressão no setor (\%) & 5,48 & 7,91 & 5,56 & 6,32 \\
$\mathcal{E}$ Vazão no setor (\%) & 19,15 & 18,70 & 13,48 & 17,11 \\
$\mathcal{E}_{\text {Pressão }}$ na linha lateral (\%) & 5,50 & 7,91 & 10,38 & 7,93 \\
$\mathcal{E}$ Vazão na linha lateral (\%) & 19,16 & 18,71 & 12,25 & 16,71 \\
\hline
\end{tabular}

Observa-se também que a máxima variação média de vazão nos setores foi igual a 19,16\%, valor próximo ao limite recomendado por KELLER \& KARMELI (1974), que é de $20 \%$.

Nos setores 1; 2 e 3, foram encontrados para o expoente de descarga, X, valores entre 0,57 e 0,60 (Tabela 2), correspondendo a regime de escoamento laminar.

TABELA 2. Parâmetros da uniformidade de irrigação e eficiência de aplicação.

\begin{tabular}{crrrr}
\hline Índices Avaliados & Setor 1 & Setor 2 & Setor 3 & Média \\
\hline CU (\%) & 97,68 & 95,23 & 93,14 & 95,35 \\
CUP (\%) & 96,87 & 98,61 & 97,75 & 97,74 \\
EA (\%) & 87,91 & 85,71 & 83,83 & 85,82 \\
CVT & 0,05 & 0,07 & 0,05 & 0,06 \\
CUE (\%) & 95,09 & 93,11 & 94,91 & 94,37 \\
CVH & 0,01 & 0,02 & 0,01 & 0,01 \\
CVE & 0,05 & 0,07 & 0,05 & 0,06 \\
X & 0,57 & 0,60 & 0,59 & 0,59 \\
\hline
\end{tabular}

Os coeficientes de variação devido a cargas hidráulicas $(\mathrm{CVH})$ foram baixos, oscilando entre 0,01 e 0,02 , enquanto o coeficiente de variação total de vazão (CVT) variou de 0,05 a 0,07 , podendo ser classificado como bom pela ABNT (1987) (Tabela 2). ZANINI et al. (1998), avaliando um sistema 
de irrigação por microaspersão em área de 81,6 ha, encontraram CVT de 0,085 a 0,11. O coeficiente de variação estatístico do microaspersor (CVE) variou de 0,05 a 0,07 (Tabela 2). Estes valores são considerados baixos, caracterizando um bom coeficiente de fabricação dos microaspersores. Os valores dos coeficientes de uniformidade $(\mathrm{CU})$ dos setores avaliados foram elevados, com base na classificação da ASAE (1996). ABREU et al. (1987) consideram, como limites aceitáveis de funcionamento, valores acima de 80\%. SOUSA et al. (1998), trabalhando com sistema de microaspersão, encontraram coeficiente de uniformidade de 82\%, enquanto ALMEIDA (1997) encontrou coeficientes altos, entre 91 e 97\%. Os coeficientes de uniformidade de pressão (CUP) também podem ser considerados altos, variando de 96,87 a 97,75\% (Tabela 2), superiores ao valor mínimo recomendado de $80 \%$, segundo a ASAE (1996). O coeficiente de uniformidade estatístico (CUE) na unidade operacional oscilou de 93,11 a 95,09\%. BARRETO FILHO et al. (2000) encontraram valores médios de CUE variando entre 89 e $92 \%$. A eficiência de aplicação da irrigação (EA) variou de 85,71 a 87,91\%. KELLER \& BLIESNER (1990) recomendam valores acima de $80 \%$.

\section{Manejo do sistema de irrigação}

O coeficiente médio de lixiviação gerado pelo agricultor, durante os primeiros 45 dias de experimento, foi de 1,21 , com desvio-padrão de 0,45 , sendo $40 \%$ menor que o sugerido inicialmente (para o período anterior à ocorrência da precipitação intensa). Na Figura 1, são apresentadas as lâminas aplicadas, a evapotranspiração real, bem como os coeficientes de lixiviação efetivamente adotados. Pode-se observar que os coeficientes de lixiviação reais diminuem durante o experimento, particularmente devido à limitada capacidade financeira do agricultor em manter elevadas taxas de irrigação. Valores de Cx inferiores a 1 indicam a não-ocorrência de lixiviação.

O balanço entre as lâminas aplicadas (por irrigação e precipitação) e a evapotranspiração está apresentado na Figura 2. Os gradientes de potencial total foram calculados para uma seção situada no topo (profundidade $35 \mathrm{~cm}$ ), e na parte inferior do perfil do solo (profundidade de $110 \mathrm{~cm}$ ). Na Figura 2 , são apresentados os gradientes, com média de 1,14, moda de 1,20 e desvio-padrão igual 0,21. Podese notar a predominância de gradientes positivos, os quais geram fluxos de lixiviação. O potencial matricial variou de $-0,20 \mathrm{~m}$ a $-0,05 \mathrm{~m}$.

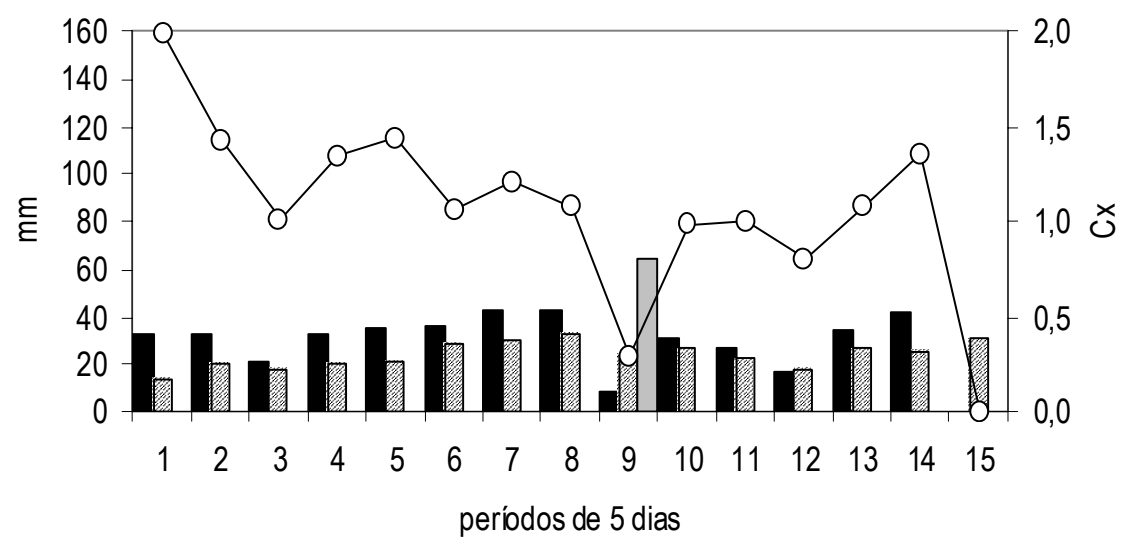

lâminas aplicadas

FIGURA 1. Lâminas aplicadas, estimativas de evapotranspiração real (ETR), precipitação (P) e Cx. 


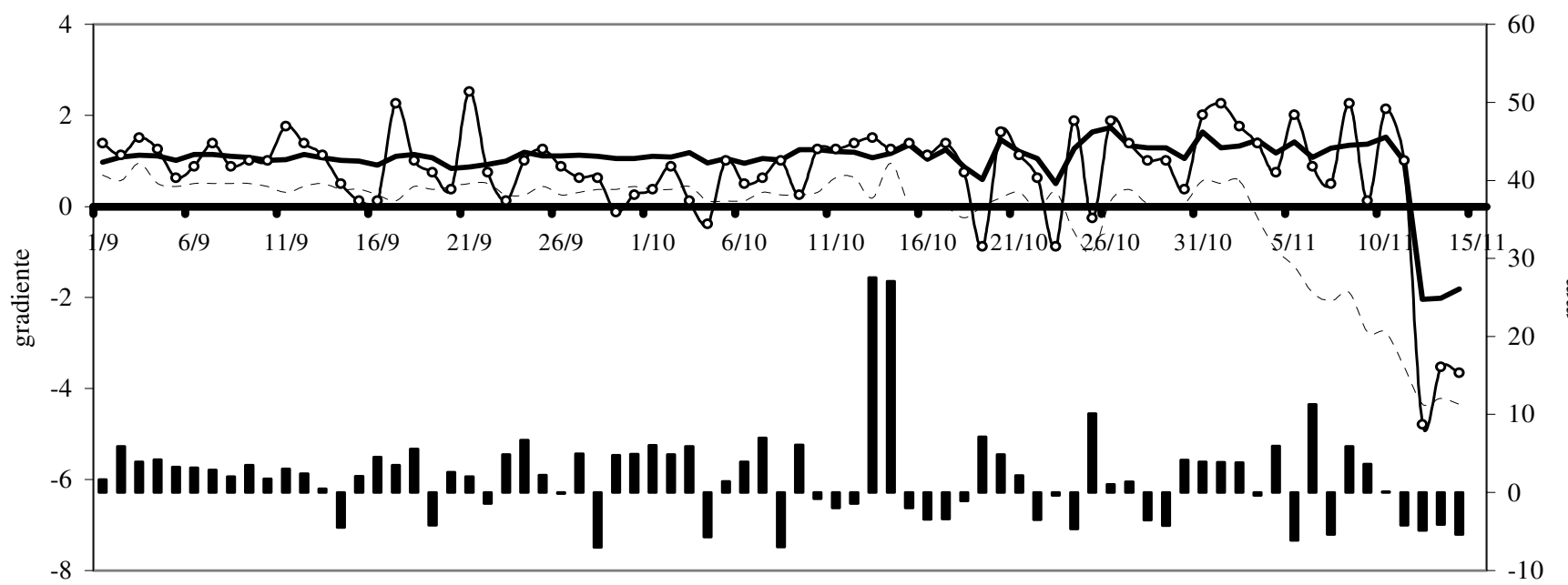

ENTRADAS-SAIDAS —- - - - - 100-40-120 média

FIGURA 2. Distribuição dos gradientes de potenciais totais de água do solo durante o experimento.

Comparando-se as hidrógrafas dos poços de observação monitorados (Figura 3), no período anterior à ocorrência de precipitação intensa, pode-se observar que os níveis de água no poço de controle apresentaram tendência linear, com coeficiente de determinação de 0,91 , e com rebaixamento aproximado de $9,6 \mathrm{~mm} \mathrm{dia}^{-1}$, devido à drenagem natural do aqüífero aluvial. Para o poço de observação localizado na área irrigada, notam-se oscilações de nível devido à lixiviação. Pode-se constatar que a irrigação produziu lâminas de recarga ao lençol, o qual apresentou coeficiente angular da regressão de $6,6 \mathrm{~mm}$ dia $^{-1}$ para seu rebaixamento. Tomando-se a diferença entre coeficientes angulares, obtém-se um valor de $3 \mathrm{~mm} \mathrm{dia}^{-1}$. De modo a inferir a lâmina de recarga, faz-se necessário estimar a porosidade efetiva do aquífero. YOUNGER (1983) sugere que a porosidade efetiva de aquíferos aluviais é de cerca de $54 \%$ da porosidade total (avaliada em 0,44 para a área em questão), o que fornece uma estimativa de 0,24 para a porosidade efetiva. Multiplicando-se esta última pela diferença entre coeficientes angulares, chega-se a uma estimativa de $0,70 \mathrm{~mm}$ dia $^{-1}$ de recarga ao lençol devida à lixiviação.

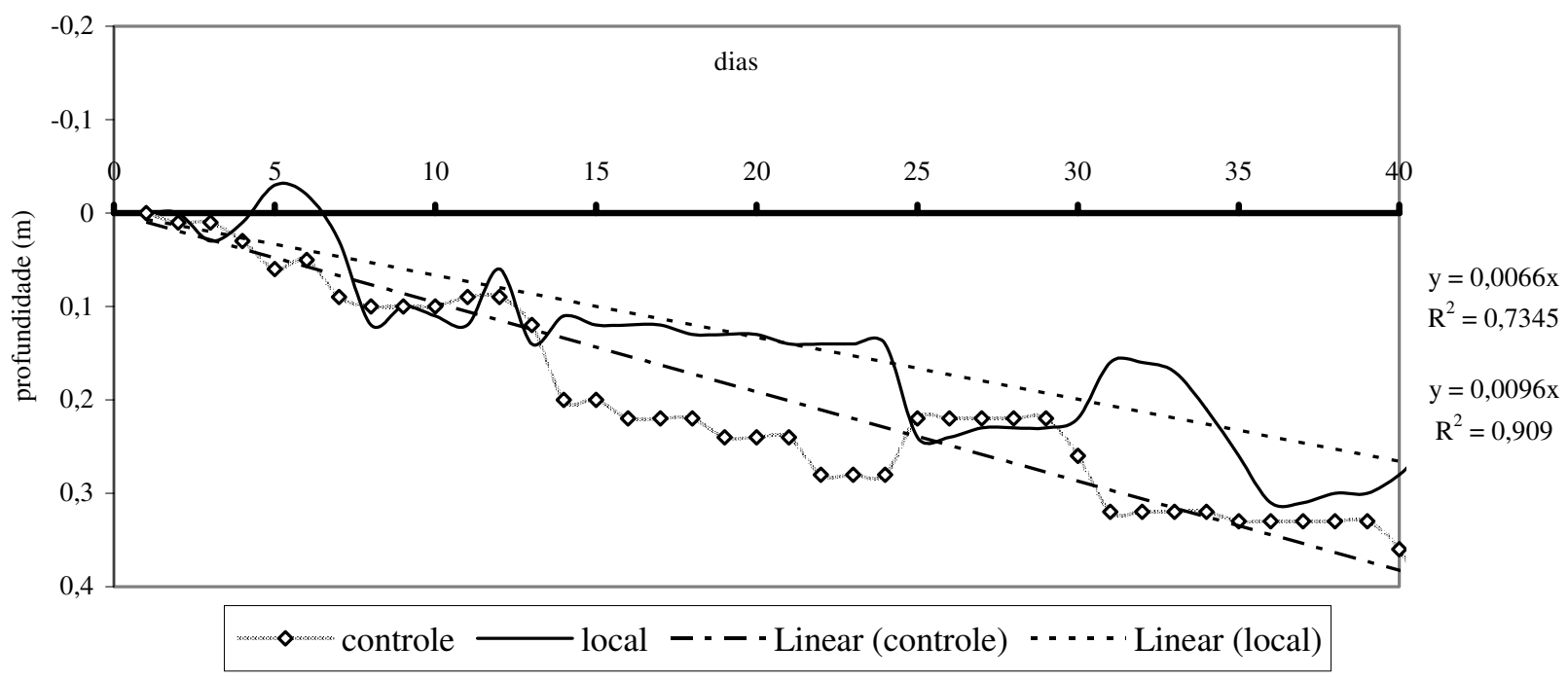

FIGURA 3. Variação de nível freático em poço de observação de controle e no local de experimento. 
Alternativamente à análise das hidrógrafas dos poços de observação, a ocorrência de fluxos descendentes no perfil pode ser analisada aplicando-se a equação de Darcy nas camadas do perfil monitoradas por tensiômetros. A precipitação pluviométrica tem relevante impacto na geração de recarga subterrânea. Embora a irrigação produza lavagem contínua do perfil, para os períodos finais de experimento, nota-se o aparecimento de fluxos ascendentes na zona de 100 a $120 \mathrm{~cm}$. As condutividades hidráulicas foram calculadas segundo a eq.(1). Análises de sensibilidade indicaram que os testes subestimaram o parâmetro $\alpha$, tendo-se utilizado um valor de $13 \mathrm{~m}^{-1}$ na estimativa dos fluxos. Os fluxos estimados para a camada de 100 a $120 \mathrm{~cm}$ apresentaram mesma ordem de grandeza daqueles calculados a partir da análise de hidrógrafas, com valor médio de $0,69 \mathrm{~mm} \mathrm{dia}^{-1}$.

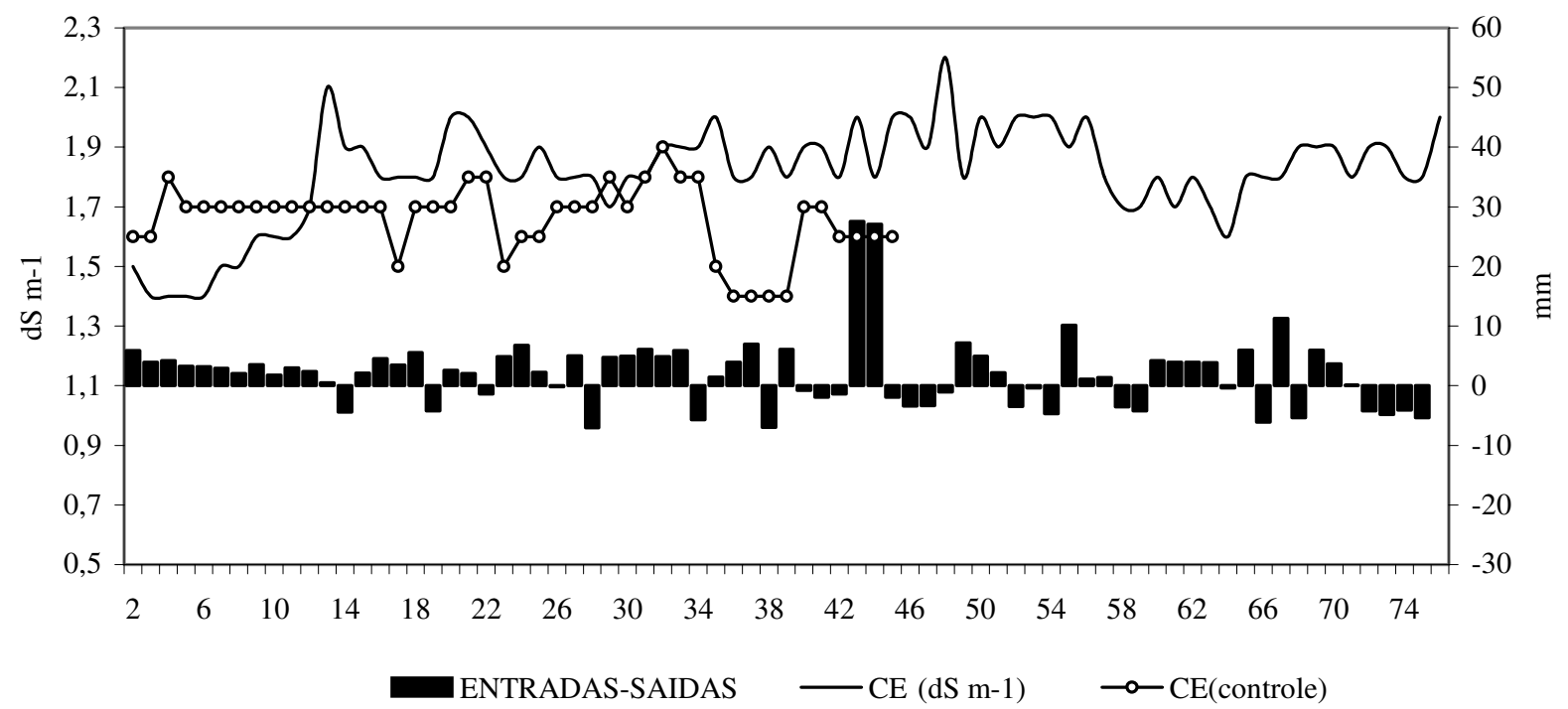

FIGURA 4. Condutividade elétrica da zona saturada e balanço entre entradas e saídas no perfil do solo.

Constatada a contribuição da irrigação para o lençol freático, deve-se verificar o comportamento da condutividade elétrica na zona saturada durante o experimento. Na Figura 4, apresenta-se a evolução da salinidade nos dois poços de observação monitorados. Nota-se gradual incremento na condutividade elétrica do lençol na área irrigada durante os 13 primeiros dias de experimento, seguido de uma estabilização que se mantém até cerca de 10 dias após a ocorrência da chuva intensa, verificando-se, a partir daí, uma diluição dos sais devida à precipitação.

Na Tabela 3, são apresentadas a condutividade elétrica e a PST do extrato saturado do solo, no início e ao final do experimento. O manejo da irrigação adotado pelo agricultor promoveu a lavagem dos sais, acarretando apenas leve aumento da condutividade elétrica (CE) do extrato nas camadas de 30 a $60 \mathrm{~cm}$ e de 60 a $90 \mathrm{~cm}$, bem como da porcentagem de sódio total (PST), mesmo utilizando água de elevada salinidade. A irrigação não promoveu a salinização nem a sodificação do solo, pois os valores de CE e PST foram menores de $4 \mathrm{dS} \mathrm{m}^{-1}$ e $15 \%$, respectivamente.

TABELA 3. Condutividade elétrica (CE) do extrato de saturação e porcentagem de sódio total (PST).

\begin{tabular}{cccrc}
\hline $\begin{array}{c}\text { Profundidade } \\
(\mathrm{cm})\end{array}$ & Início & FE $\left(\mathrm{dS} \mathrm{m}^{-1}\right)$ & Início & PST (\%) \\
\hline $0-30$ & 0,71 & 0,86 & 6,48 & 7,48 \\
$30-60$ & 1,64 & 1,70 & 11,84 & 12,98 \\
$60-90$ & - & 1,70 & - & 13,11 \\
\hline
\end{tabular}




\section{CONCLUSÕES}

A vazão de sistema foi mantida próxima à indicada pelo fabricante, embora a pressão de operação dos microaspersores estivesse levemente alterada.

Os coeficientes de uniformidade de irrigação dos setores avaliados foram altos, mostrando que o sistema foi hidraulicamente bem dimensionado, possuindo microaspersores de boa qualidade e operando adequadamente.

A eficiência de aplicação nos setores avaliados foi considerada boa, caracterizando uma adequada qualidade na irrigação.

As variações de vazão e pressão não foram altas, a ponto de comprometer o manejo.

Os fluxos na zona não-saturada foram predominantemente descendentes, indicando ocorrência de lixiviação do perfil do solo.

O coeficiente médio de lixiviação $(1,21)$ aplicado pelo pequeno agricultor foi suficiente para promover a lavagem do perfil do solo e garantir a manutenção do equilíbrio salino, tanto na zona radicular, quanto na zona saturada, podendo ser utilizado na microaspersão em áreas aluviais com circulação hídrica adequada, onde a condutividade hidráulica saturada é da ordem ou superior a $10^{-6} \mathrm{~m} \mathrm{~s}^{-1}$, mesmo para água de irrigação com condutividade elétrica da ordem de $1,40 \mathrm{dS} \mathrm{m}^{-1}$, e com culturas de similar tolerância à salinidade.

\section{AGRADECIMENTOS}

Ao "Programa Universidade Solidária" (UNISOL)/MEC, pelo apoio financeiro na implantação do sistema de irrigação. À CAPES, pela concessão da bolsa de pesquisa. À UFRPE, pelo apoio concedido na realização desta pesquisa. Aos revisores do trabalho, pelas valiosas contribuições.

\section{REFERÊNCIAS}

ASSOCIAÇÃO BRASILEIRA DE NORMAS TÉCNICAS. Projeto 12: 02-08-20. Sistema de irrigação localizada: Avaliação do desempenho. Rio de Janeiro, 1987. 11 p.

ABREU, J.M.H.; LOPES, J.R.; REGALADO, A. P.; HERNANDEZ, J.F.G. El riego localizado. Madrid: Instituto Nacional de Investigações Agrárias, 1987. 317 p.

ALMEIDA, F.T. Avaliação dos sistemas de irrigação pressurizados e do manejo da água na cultura da banana no Projeto Gorutuba. 1997. 96 f. Dissertação (Mestrado em Irrigação e Drenagem) Universidade Federal de Viçosa, Viçosa - MG, 1997.

AMERICAN SOCIETY OF AGRICULTURAL ENGINEERS. Field evaluation of microirrigation Systems. St. Joseph, 1996. p. 792-7.

AYERS, R.S.; WESTCOT, D.W. A qualidade da água na agricultura. Tradução de: H.R. GHEYI, J.F. MEDEIROS, F.A.V. DAMASCENO. Campina Grande: UFPB, 1999. 153 p. (Estudos FAO: Irrigação e Drenagem, 29).

BARRETO FILHO, A.A.; DANTAS NETO, .J.; MATOS, J.A.; GOMES, E.M. Desempenho de um sistema de irrigação por microaspersão, instalado a nível de campo. Revista Brasileira de Engenharia Agrícola e Ambiental, Campina Grande, v.4, n.3, p. 309-14, 2000.

BERNARDO, S. Manual de irrigação. 6. ed. Viçosa - MG: UFV, 1996. 596 p.

BERNARDO, S. Impacto ambiental da irrigação no Brasil. In: SILVA, D.D. da; PRUSKI, F.F. (Ed.). Recursos hídricos e desenvolvimento sustentável da agricultura. Viçosa: MMA, SRH, ABEAS, UFV, 1997. 252 p. 
BONAM, B.J. Distribution patterns of microirrigation spinner and spray emitters. Applied Engineering in Agriculture, St. Joseph, v.5. n.2, p.50-6, 1989.

BRALTS, V.F.; KESNER, C. Drip irrigation field uniformity estimation. Transactions of the ASAE, St. Joseph, v.24, n.5, p.1369-74, 1983.

BRESLER, E. Analysis of trickle irrigation with application to design problems. Irrigation Science, Amsterdam, v.1, n.1, p.3-17, 1978.

COMPANHIA INTEGRADA DE SERVIÇOS AGROPECUÁRIOS (CISAGRO). Projeto de irrigação da Fazenda Nossa Senhora do Rosário. Pesqueira, 1990. 120 p.

DOORENBOS, J.; KASSAN, A.H. Efectos del água en al rendimiento de los cultivos. Roma: FAO, 1979. 212 p. (Riego y Drenage, 33)

DOORENBOS, J.; PRUITT, W.O. Las necessidades de água de los cultivos. Roma: FAO, 1976. 143 p. (Riego y Drenage, 24)

EMPRESA BRASILEIRA DE PESQUISA AGROPECUÁRIA. Manual de métodos de análise de solos. Rio de Janeiro, 1979. 247 p.

EMPRESA BRASILEIRA DE PESQUISA AGROPECUÁRIA. Serviço Nacional de Levantamento e Conservação dos Solos. Manual de métodos de análise de solos. 2. ed. Rio de Janeiro, 1997. 212 p.

GARDNER, W.R. Some steady state solutions of the unsaturated moisture flow equation with application to evaporation from water table. Soil Science, Baltimore, v.85. p.228-32, 1958.

IBGE. 1996. Censo Agropecuário 1995-1996. Disponível em: <http:/ www.incra.gov.br/sade> Acesso em: 4 março 2002.

KELLER, J.; BLIESNER, R.D. Sprinkler and trickle irrigation. New York: van Nostrand Reinhold, $1990.625 \mathrm{p}$.

KELLER, J.; KARMELI, D. Trickle irrigation design parameters. Transactions of the ASAE, St. Joseph, v.17, n.4, p.678-84, 1974.

KELLER, J.; KARMELI, D. Trickle irrigation design. Glendora: Rain Bird Sprinklers Manufacturing Corp., 1975. 133 p.

LIBARDI, P.L. Propriedades físico-hídricas do solo no estudo do manejo e da conservação do solo e da água. In: OLIVEIRA, T.S.; ASSIS, R.N.; ROMERO, R.E.; SILVA, J.R.C. Agricultura, sustentabilidade e o semi-árido. Fortaleza: UFC, 2000. p.214-23.

MASS, E.V.; HOFFMAN, G.J. Crop salt tolerance: current assessment. Journal of Irrigation and Drainage Division, New York, v.103, n.1, p.115-34, 1977.

MATOS, J.A.; DANTAS NETO, J.; AZEVEDO, H.M.; AZEVEDO, C.V.A. Características hidráulicas do microaspersor Dan Sprinkler. Irriga, Botucatu, v.1, n.3, p.30-44, 2001.

MERRIAN, J. L.; KELLER, J. Farm irrigation systems evaluation: A guide for management. Logan: Agricultural and Irrigation Engineering Department, Utah State University, 1978. 271 p.

MONTENEGRO, A.A.A. Relatório Técnico das Atividades no Controle da Salinidade de Água e Solo em Pequenos Perímetros irrigados. Recife: Banco do Nordeste, Universidade Federal Rural de Pernambuco, 2000. 8 p.

REYNOLDS, W.D.; ELRICK, A.; CLOTHIER, B.E. The constant head well permeameter: effect of unsaturated flow. Soil Science, Baltimore, v.139, n.2, p.172-80, 1985. 
RIBEIRO, M.R.; FREIRE, F.J.; MONTENEGRO, A.A.A. Solos halomórficos no Brasil: ocorrência, gênese, classificação, uso e manejo sustentável. In: Tópicos em Ciência do Solo. Viçosa - MG: Sociedade Brasileira de Ciência do Solo, 2000. v.1, p.165-208.

SANTIAGO, F. dos S. Efeito do manejo da irrigação na salinidade e umidade de neossolo flúvico, com a cultivar de repolho midori (Brassica oleracea var. capitata), no município de Pesqueira -PE. 2002. 115 f. Dissertação (Mestrado em Ciência do Solo) - Universidade Federal Rural de Pernambuco, Recife, 2002.

SOUSA, V.F.; FOLEGATTI, M.V.; ARAGÃO, E.C.; REBELO, A.L.F.; BASTOS, E.A. Uniformidade de distribuição de água em um sistema de irrigação por microaspersão instalado numa área cultivada com banana. In: CONGRESSO BRASILEIRO DE ENGENHARIA AGRÍCOLA, 27. 1998, Poços de Caldas. Anais...Poços de Caldas: Sociedade Brasileira de Engenharia Agrícola, 1998, v.1. p.211-13.

YOUNGER, P. L. Simple generalized methods for estimating aquifer storage parameters. Quarterly Journal of Engineering Geology, Geological Society, Londres, v.26, n.1, p.127-35, 1993.

ZANINI, J.R.; PAVANI, L.C.; TAROZZO, M. Avaliação da vazão de um sistema de irrigação por microaspersão em cultura de bananeira. In: CONGRESSO BRASILEIRO DE ENGENHARIA AGRICOLA, 27., 1998. Poços de Caldas. Anais... Poços de Caldas: Sociedade Brasileira de Engenharia Agrícola, 1998. v.2, p.290-2. 